\title{
El dominio conceptual del deporte para la construcción de metáforas deslegitimadoras en el discurso de la extrema derecha española
}

\section{The conceptual domain of sport in the formation of delegitimating metaphors in the Spanish ultraconservative discourse}

\author{
MARINA DÍAZ-PERALTA \\ GRACIA PINERO-PINERO \\ Universidad de Las Palmas de Gran Canaria. \\ Instituto Universitario de Análisis y Aplicaciones Textuales. España \\ marina.diazperalta@ulpgc.es \\ Universidad de Las Palmas de Gran Canaria. \\ Instituto Universitario de Análisis y Aplicaciones Textuales. España. \\ Correo electrónico: gracia.pinero@ulpgc.es
}

Partiendo de un marco teórico constituido por la teoría de la metáfora conceptual (Lakoff y Johnson 1980), el paradigma narrativo de Fisher $(1984,1985)$ y conceptos procedentes del análisis crítico del discurso (Van Dijk 2011), este trabajo analiza el modo en que el discurso político categoriza mediante metáforas conceptuales un hecho complejo que tuvo lugar en la transición española: la legalización del Partido Comunista de España. Veremos cómo la prensa de extrema derecha, contraria a esta decisión, enmarca la noticia en un sistema de conocimientos, actitudes, creencias y juicios de valor propio de la comunidad a la que se dirige recurriendo a una metáfora continuada cuyo marco fuente es el DEPORTE — y, concretamente, el submarco del FútBOL—y a una serie de procesos cognitivos generadores de una narrativa que teje la historia de una traición. Estos mecanismos contribuyen a construir la identidad discursiva de los protagonistas de este episodio y, con ello, a la deslegitimación de las actuaciones que dieron como resultado la inscripción de ese partido en el Registro de Asociaciones Políticas.

Palabras clave: metáfora, discurso político, ideología, narrativa, dominio DEPORTE

Starting from a theoretical framework which uses the Conceptual Metaphor Theory (Lakoff y Johnson 1980), the Narrative Paradigm (Fisher 1984, 1985) and concepts taken from Critical Discourse Analysis (Van Dijk 2011), this paper examines the way in which political discourse categorize through 
conceptual metaphors a complex event such as the legalization of the Spanish Communist Party, which occurred during the period of the Spanish Transition. It can be seen how the ultraconservative press, whose ideology was against the legalization, places the news within their readership's system of knowledge, beliefs and judgments. The author achieves this using a continued metaphor whose source frame is SPORTS, particularly FOOTBALL subframe, and a series of cognitive processes that generate a mini-narrative that tells the story of treason. These mechanisms contribute to the establishing of the discursive identity of the protagonists of this event and subsequently to the delegitimization of what happened as a result of the inclusion of this party in the Register of Political Associations.

Key words: metaphor, political discourse, ideology, narrative, sPORT domain

\section{Marco CONCEPTUAL}

Este artículo pretende ser una muestra de cómo el discurso político de la extrema derecha plasma su postura ideológica ante un episodio trascendental de la historia contemporánea española: la legalización el 9 de abril de 1977 del Partido Comunista de España (PCE). Para ello, haremos uso del editorial del diario El Alcázar publicado dos días después, en el que tal acontecimiento y las circunstancias que lo rodearon quedan representados mediante metáforas conceptuales que se perfilan sobre un dominio cognitivo especialmente arraigado en el país: el deporte; más concretamente, sobre las acciones propias de un partido de fútbol, espectáculo deportivo omnipresente en los medios de comunicación y en la vida diaria.

Decíamos que la metáfora, modelo cognitivo idealizado (Lakoff 1987), es el instrumento elegido por el autor del editorial para construir la columna vertebral que sostiene su narración de principio a fin. Por ello, nuestro análisis precisa un marco conceptual, que seguidamente desarrollaremos, compuesto a partir de constructos complementarios. Así, además de la teoría de la metáfora conceptual (Lakoff y Johnson 1980, 1999; Johnson 1987, 1993; Lakoff 2008; Lakoff y Turner 1989; Turner 1991) y sus vínculos con las nociones de marco (Fillmore 1985), dominio (Langacker 1987) y esquema de imagen (Johnson 1987), son herramientas esenciales en nuestro caso el paradigma narrativo de Fisher $(1984,1985)$ y el concepto de modelo mental (Van Dijk 2011: 21), procedente del análisis crítico del discurso, corriente que se ocupa del papel que juegan los medios de comunicación en el mantenimiento, legitimación y refuerzo del orden vigente (Casero 2007).

Dice MacIntyre: "man is [...] essentially a story-telling animal" (MacIntyre $198 \mathrm{I}$ : 20I). De esta idea parte Fisher $(1984,1985)$ para proponer su paradigma narrativo, que entiende que una buena historia consigue, en mayor medida que un buen argumento, una comunicación eficaz de la realidad, pues es siempre más persuasiva. Con independencia de su forma, las narraciones son historias que se desarrollan en la interacción social para dotar al mundo de un sentido; por ello, afirma Fisher (1984, 1985), comprender el significado y la relevancia de la vida en toda su dimensión requiere el reconocimiento de su estructura 
narrativa; esto es, de las actitudes, ideologías, normas y valores que sustentan las decisiones humanas y la vida en comunidad. Cada grupo social tiene, por consiguiente, su propia narrativa, que implica un compromiso ético y moral (Glenister 2004). Y es esta la que utilizan y moldean, para conectar con sus lectores, los medios de comunicación, que, como subraya Grossi (2004), asumen una posición central en la mediación simbólica de las experiencias sociales dando forma con su discurso a significados, sentidos e imágenes sobre la realidad, que pasan a ser compartidos por una colectividad.

A pesar de esto, la metáfora conceptual, creadora también de imágenes, sentidos y significados, ha sido largamente olvidada en los estudios sobre las narraciones que subyacen a los discursos políticos (Hart 2008). Charteris-Black (2004) y Chilton (2005) afirman que esta debe ser un centro de interés para tales investigaciones, en tanto que también conforma un modo coherente de contar la realidad. La metáfora es una de las estructuras ideológicas del discurso, constructora de una narrativa que impone una determinada comprensión de la realidad en detrimento de otras (Chilton 1996; Van Dijk 2006).

Este mecanismo de categorización, basado en el emparejamiento de dos dominios cognitivos, permite conceptualizar realidades complejas o abstractas en términos de otras más sencillas, más concretas y, por ello, más asequibles. El resultado es un isomorfismo o "reconocimiento de un conjunto de relaciones comunes en el seno de entidades diferentes" (Millán \& Narotzky 1986: 16-17) que contribuye a explicar o esclarecer una determinada esfera de la experiencia.

Por otra parte, la metáfora puede emplearse para subrayar aquellos aspectos que se pliegan a un ideario ${ }^{1}$ y que resultan en un cuerpo conceptual coherente, revelador de la ideología del grupo social que las genera, mediante la aplicación de una serie de procesos cognitivos. Entre estos últimos destacan la elección de dominios fuente, el establecimiento de proyecciones entre los dominios fuente y meta y la asignación de roles semánticos (Pinero-Pinero, Díaz-Peralta \& García-Domínguez 2014; Piñero Piñero \& Moore 2014; Díaz-Peralta 2016, 2018). Según explica Musolff, la metáfora activa:

[...] a set of assumptions made by competent members of a discourse community about 'typical aspects of a source-situation', for example, its participants and their roles, the 'dramatic' storylines and outcomes and conventional evaluations of whether they count as successful or unsuccessful, normal or abnormal, permissible or illegitimate, etc. (Musolff 2009: 28).

Los términos marco (Fillmore 1985, 2006; Fillmore y Atkins 1992) y dominio (Langacker 1987) surgen en el ámbito de la semántica cognitiva y establecen que las categorías no constituyen unidades aisladas, sino que han de ser comprendidas tomando

\footnotetext{
${ }^{1}$ En este sentido, Paivio dice que "Metaphor is a solar eclipse. It hides the object of study and at the same time reveals some of its most salient and interesting characteristics when viewed through the right telescope" (Paivio 1979: 150).
} 
en consideración un contexto estructurado de experiencias, creencias o prácticas. Consecuentemente, ambos términos designan un sistema de conceptos de tal modo relacionados que para entender uno de ellos es necesario comprender toda la estructura en la que se inserta (Croft \& Cruse 2004: 8). En palabras de Lakoff:

Frames are the mental structures that shape the way we view the world. Frames are the connotations that we instil words with and the context in which we place those value-laden words. No one sees or hears frames, they are part of what scientists refer to us the cognitive unconscious, inaccessible to the conscious mind, but at play in our decisions, our actions and the way that we process data. All words are defined relative to conceptual frames. When we hear a word its frame or collection of frames is activated in our brain (Lakoff 2004: 4).

Las palabras, por tanto, constituyen el nodo de acceso (Langacker 1987: 163) a toda elección de marcos o dominios fuente que lleva a cabo el emisor para categorizar metafóricamente el tópico del discurso está condicionada tanto por su realidad experiencial como por las representaciones sociales compartidas (Meyer 2001:21) de los acontecimientos narrados. De este modo, la metáfora actualiza el relato de los eventos políticos en un sistema de conocimientos, actitudes, creencias y juicios de valor propio de la comunidad (Van Dijk 2002: 208) a la que va dirigido.

Cierra nuestro marco teórico el ya mencionado concepto de esquema de imagen (Lakoff \& Johnson 1980; Johnson 1987), ese patrón dinámico y recurrente de la cognición humana, producto de nuestra interacción social y corporal con el entorno y construido a partir de "our perception, bodily movement through space, and physical manipulation of objects" (Johnson 1987: 23). Estos patrones proporcionan una estructura coherente y significativa para comprender aspectos abstractos de la realidad y aportan el dominio fuente de metáforas estructurales e imago-esquemáticas, que se prestan a razonamientos complejos (Ruiz de Mendoza \& Otal 2002; Ruiz de Mendoza \& Pérez 2011).

\section{ANÁLISIS}

Con estos planteamientos teóricos hemos desarrollado un análisis del editorial ya mencionado de acuerdo con la siguiente metodología. En primer lugar, hemos leído detenidamente el texto con el fin de hacer una primera identificación (Stefanowitsch 2006: 2-11) de todas las instanciaciones lingüísticas de metáforas conceptuales relacionadas tanto con la legalización del PCE como con sus actores protagonistas: el gobierno, los órganos judiciales consultados y los sectores del pueblo español partidarios de la legalización; en segundo lugar, para garantizar que las estructuras seleccionadas responden efectivamente a un uso metafórico hemos aplicado el siguiente procedimiento, basado en la propuesta metodológica de Steen et al. (Steen et al. 2010: 14-15): 
1. Se han identificado las unidades léxicas que permiten inferir la instanciación del dominio o marco fuente objeto de interés: EL DEPORTE.

2. Se ha determinado el significado contextual de cada una de las unidades léxicas identificadas; es decir, se ha analizado el modo en que cada una de ellas se aplica a una entidad, relación o característica de la situación evocada por el cotexto.

3. Se han considerado metafóricas todas aquellas unidades cuyo significado contextual se puede entender en relación con un significado básico más concreto, o más antiguo, o relacionado con la experiencia corpórea.

Resultado de este examen fue la delimitación de un corpus constituido por las siguientes metáforas: LA LEGALIZACIÓN DEL PCE ES UN GOL EN NUESTRA PROPIA PORTERÍA, LA NO LEGALIZACIÓN DEL PCE ES LA PORTERÍA DEL GOBIERNO, LA ESTRATEGIA DEL GOBIERNO ES ECHAR BALONES FUERA, LA ESTRATEGIA DEL GOBIERNO ES JUGAR A OTRA BANDA, LA LEGALIZACIÓN DEL PCE ES UN BALÓN EN JUEGO, LOS PARTIDARIOS DE LA LEGALIZACIÓN SON HINCHAS y, finalmente, LOS PARTIDARIOS DE LA LEGALIZACIÓN SON FIERAS. En todas ellas la incompatibilidad semántica con el contexto (Le Guern 1976: 19) orienta al lector hacia la interpretación pretendida por quien escribe.

A partir de este corpus, hemos observado los procesos cognitivos que contribuyen a construir la identidad discursiva de los protagonistas de los acontecimientos narrados por el editorial y, con ello, como demostraremos, a la deslegitimación (Van Dijk 1998) de sus actuaciones, que dieron como resultado la inscripción del PCE en el Registro de Asociaciones Políticas.

Como es sabido, tras la muerte de Franco, algunos sectores del régimen lograron, con el apoyo de la monarquía, una progresiva reforma del sistema político a partir de la evolución de las leyes y de las instituciones franquistas. El éxito de esta reforma, liderada por Adolfo Suarez, exigía, por un lado, neutralizar la resistencia de los inmovilistas del régimen; y, por otro, atraer a una buena parte de la oposición democrática. Con este fin, Suárez propuso la Ley de Reforma Política, promulgada el 4 de enero de 1977, que reconocía los derechos fundamentales de los españoles, confería la potestad legislativa en exclusiva a la representación popular y preveía un sistema electoral democrático, que se materializaría en las elecciones celebradas en junio de 1977. Con la aprobación de esta ley se crearon las condiciones necesarias para la legalización de los partidos políticos con la sola excepción del Partido Comunista de España. La oposición frontal del búnker, defensor del mantenimiento de la legalidad franquista, y de amplios sectores del ejército hizo que la legalización del PCE se convirtiera en un auténtico escollo para el proceso democrático. Ahora bien, el cambio en los estatutos del partido y su reacción pacífica ante el asesinato de los abogados laboralistas de izquierdas a manos de ultraderechistas en lo que se ha denominado la matanza de Atocha provocaron que Suárez lograra su legalización.

No todas las publicaciones españolas acogieron la noticia de la misma manera. Así, en periódicos como Informaciones o El País aparecían editoriales y artículos de opinión cuyos autores se congratulaban pues entendían que este hecho garantizaba la democracia. 
En este sentido, el editorial de El País del día 10 de abril califica la inscripción del PCE en el Registro de Asociaciones Políticas de buena noticia "porque contribuirá a clarificar el ambiente político y a normalizar la situación cara a las elecciones, que podrán celebrarse en un clima de pluralismo real". En Informaciones, por su parte, Abel Hernández afirma, en la edición del día 11 de abril, que "Al fin se ha impuesto el sentido común y el sentido político".

Frente a esta postura exhibida por ambos diarios, otras publicaciones periódicas de distinta ideología manifestaban su alarma sin ambages. Este es el caso del editorial del $A B C$, publicado también el día 10 de abril, donde se puede leer lo siguiente: "sin ningún afán de dramatizar entendemos que esta es una gravísima decisión y un error de nuestros gobernantes del que sólo deseamos que no tengan que arrepentirse los hombres que han tomado sobre sí tamaña responsabilidad".

Del mismo modo, la prensa de extrema derecha muestra su indignación ante este acontecimiento. Un claro ejemplo de ello es el editorial en el que se centra nuestro análisis, aparecido, como ya hemos explicado, el 11 de abril en El Alcázar. Este periódico, fundado en 1936 durante el asedio del Alcázar de Toledo, se convirtió a lo largo de la Guerra Civil en el diario del frente de Madrid. Tras numerosas dificultades económicas y cambios en la dirección que lo fueron llevando hacia una postura independiente y aperturista dentro del franquismo, en 1968 pasó a manos de la Prensa del Movimiento y regresó a la línea más dura del régimen. En 1975, el periódico fue adquirido por la Confederación Nacional de Hermandades de Excombatientes y, tras la muerte de Franco, se convirtió en vehículo de expresión del búnker hasta el punto de que, en sus páginas, llegó incluso a arengar a sus lectores a favor de un golpe de estado (Muñoz 1986; Montero, Rodríguez-Virgili \& GarcíaOrtega 2008; Zugasti 2008).

Afirma Lakoff que "An important conclusion of research in cognitive studies is that moral thinking is imaginative and that it depends fundamentally on metaphorical understanding" (Lakoff 2002: 47). Precisamente, nuestro texto se articula sobre la base de una metáfora continuada, que da cuerpo a la representación simbólica de unos acontecimientos políticos de enorme trascendencia social. Haciendo uso de este procedimiento cognitivo, con intención deslegitimadora, el periódico se constituye, como tendremos ocasión de comprobar, en un actor político (Casero 2004) que persigue ejercer su influencia en la opinión pública y crear una identidad negativa del colectivo partidario de la legalización del PCE. Como afirma Casero: "Los discursos y, especialmente, el procedente de los medios, se convierten así en formas de representación identitaria que ordenan, organizan e influyen en nuestra interpretación del mundo" (Casero 2007: 71-72).

Para construir esta metáfora continuada el productor textual acude al dominio o marco fuente DEPORTE, cuya importancia para conceptualizar los hechos políticos ha sido puesta de relieve por distintas investigaciones (entre otras, Beer \& De Landtsheer 2004; McEntee-Atalianis 2011, 2013). En nuestro texto, el marco del deporte se concreta en un submarco; esto es, una categoría subordinada que denota una parte del evento complejo significado por el marco (Fillmore \& Atkins 1992: 80). En efecto, el editorial selecciona el 
submarco específico y cotidiano del fútвol, que se activa desde el mismo título del artículo, GOL, y que se materializa en sus primeras líneas con otros sustantivos como gradas e hinchas:

El partido Comunista ha sido legalizado por el Gobierno. En las gradas ocupadas por sus hinchas (los del Partido y los del Gobierno), el grito de alegría ha sonado como un rugido: ;Gol!

El fútbol constituye un modelo mental (Van Dijk 2011: 21-22) frecuentemente empleado en el discurso español para representar subjetivamente los eventos de los que se habla. Su éxito, su aceptabilidad social se explica porque es una muestra evidente de la definición dada por Van Dijk de este concepto: "interfaz que combina lo personal y lo singular, por una parte, con lo social y lo compartido, por otra" (Van Dijk 2011: 22).

Este submarco genera una serie de procesos cognitivos relacionados con la actividad política y social en curso; con la distribución de roles entre los protagonistas y con las relaciones que existen entre ellos; y, por último, con las intenciones y los objetivos de los participantes. Además, supone la interpretación y la evaluación moral de los acontecimientos narrados según los intereses y el sesgo ideológicos del autor del texto (Musolff 2009).

El subdominio del fútвol resulta, como veremos, especialmente apropiado para dotar de una dimensión física y, por tanto, fácilmente perceptible, a unos acontecimientos políticos complejos, de grandes repercusiones sociales e ideológicas. Nos referimos, como hemos repetido, a los sucesos que culminan en la noticia inesperada y casi inverosímil para los españoles, sobre todo para los afectos al antiguo régimen, de que el PCE se ha inscrito en el Registro de Asociaciones Políticas. Tal y como explica Prego (2002): "La noticia cae literalmente como una bomba en el país. Provoca el estupor y el miedo en los sectores no politizados, una indignación inmensa en la derecha franquista y una furia casi incontenible en el seno del Ejército".

Son numerosas las incógnitas en torno a la actuación del presidente Suárez en este periodo y, particularmente, en relación con un proceso secreto de negociación con Santiago Carrillo — la "bestia negra del franquismo", en palabras de Prego (2002)—, que dio como resultado la legalización del PCE, imprescindible para las fuerzas democráticas y que, para el búnker y el ejército, suponía el peor final posible para los cuarenta años del régimen.

Esta difícil situación se corporeiza en nuestro editorial mediante un conjunto de proyecciones metafóricas que asignan roles, incorporan valoraciones éticas sobre lo legítimo y lo ilegítimo y conforman una narrativa cuyo hilo argumental es, según iremos viendo, la historia de una traición.

Para facilitar la interpretación de los datos proporcionados por el análisis, hemos organizado tales proyecciones atendiendo a la distinción que establecen Ruiz de Mendoza (2000: 111-113) y Ruiz de Mendoza \& Pérez (2011) entre metáforas estructurales y no estructurales. En las primeras, la complejidad del dominio fuente desencadena numerosas proyecciones ontológicas y epistémicas. Como es sabido, se denominan correspondencias ontológicas a las asociaciones entre conceptos que ponen de manifiesto las relaciones 
analógicas existentes entre las partes más relevantes de los dominios; y correspondencias epistémicas, a las proyecciones de conocimientos que permiten hacer las inferencias que fundamentan una metáfora (Lakoff \& Johnson 1980; Lakoff 1993).

En las metáforas no estructurales, por su parte, el dominio fuente solo exporta un rasgo y las correspondencias ontológicas más básicas que lo acompańan, pues su objetivo es dar prominencia a una parte del dominio meta mediante una característica relevante y quintaesencial del dominio fuente (Ruiz de Mendoza 2000: 111-113).

\subsection{Metáforas estructurales}

Los acontecimientos se describen en el editorial como un partido de fútbol en el que el equipo del que forman parte El Alcázar y sus lectores pierde el enfrentamiento porque uno de sus jugadores, proyectado sobre el Gobierno, mete el gol en su propia portería de forma absolutamente intencionada. Este escenario se encarna en distintas metáforas conceptuales subordinadas a una metáfora principal, troncal, LA LEGALIZACIÓN DEL PCE ES UN GOL EN NUESTRA PROPIA PORTERÍA, que ya se infiere desde el mismo título y que se explicita en el siguiente párrafo:

El gol lo ha metido el gobierno en su propia portería. En la portería que se juró y perjuró que estaba defendida y bien defendida contra la amenaza comunista.

Los significados que evoca esta primera metáfora troncal (LA LEGALIZACIÓN DEL PCE ES UN GOL EN NUESTRA PROPIA PORTERÍA) suponen un mecanismo de evaluación interna que pone al descubierto el menosprecio por la actuación del Gobierno, que queda deslegitimado ante el receptor del artículo, contrario, como su autor, a que obtenga carta de naturaleza en el escenario político español el partido de Santiago Carrillo, Consejero de Orden Público durante la Guerra Civil y uno de los responsables de las matanzas de Paracuellos de Jarama.

Por tanto, esta metáfora contribuye, gracias al contexto lingüístico y al cognitivo, a la creación de espacios mentales cohesionadores del grupo endógeno (Van Dijk 2005, 2011). Con su discurso, el editorial de El Alcázar"se sitúa en las bases de las prácticas sociales y de los modelos cognitivos" (Casero 2007: 72) de sus lectores - contrarios a la legalización, según hemos dicho-, quienes consideran que juegan en el mismo equipo que el Gobierno y, particularmente, que su presidente: Adolfo Suárez se ha formado con las enseńanzas y las doctrinas de la Falange y ha ocupado el cargo de Vicesecretario del Movimiento. Por lo tanto, El Alcázar y sus lectores interpretan que el presidente del Gobierno se está aplicando a la defensa de su portería, proyectada sobre la no legalización: LA NO LEGALIZACIÓN DEL PCE ES LA PORTERÍA DEL GOBIERNO.

El gol lo ha metido el gobierno en su propia portería. En la portería que se juró y perjuró que estaba defendida y bien defendida contra la amenaza comunista. 'Ni comunismo, ni separatismo, ni terrorismo', se había proclamado enfáticamente. 
El separatismo campa por sus respetos; al comunismo se le ha dado pase de libre circulación y los terroristas van saliendo, pian pianito, a la calle.

No olvidemos, además, que Suárez había manifestado con claridad su intención de oponerse a la legalización del PCE mientras no se produjeran cambios en sus estatutos, cosa que, efectivamente, acabó sucediendo. Para los inmovilistas, según iremos viendo en nuestro texto, la traición que narra el editorial consistió no solo en posibilitar la legalización, sino además en llevarla a cabo haciendo creer a la opinión pública que se trataba de una acción ineludible a la vista de los dictámenes de las instancias judiciales consultadas por el Gobierno.

Por otra parte, en la construcción de la metáfora principal (LA LEGALIZACión DEL PCE ES UN GOL EN NUESTRA PROPIA PORTERÍA) ya se descubre una pauta que se reiterará en buena parte de las analogías que a ella se subordinan: el autor configura el dominio fuente recurriendo a una diversidad de esquemas de imagen (Lakoff \& Johnson 1980; Johnson 1987), que generan metáforas idóneas para el razonamiento complejo y que se construyen con dominios fuente básicos tales como ESPACIO, ACCIÓN y FUERZA.

En el siguiente fragmento aparecen las instanciaciones lingüísticas de algunas de esas metáforas estructurales derivadas de la troncal y en cuyos marcos fuente se advierte, como enseguida precisaremos, esa confluencia que ya hemos mencionado de distintos esquemas de imagen:

Hace poco nos hemos ocupado de la táctica del Gobierno de echar balones fuera. [...]. El balón de la legalización del Partido Comunista, pasado al Tribunal Supremo. De este alto organismo le fue devuelta la pelota. Entonces el Gobierno ha jugado a otra banda. El balón ha ido al Fiscal del Reino.

Queda en el aire la pregunta de qué hubiera ocurrido si el Fiscal del Reino, en vez de atenerse a la forma, hubiera entrado en la cuestión de fondo, es decir, en si el comunismo es compatible con nuestro sistema institucional. Pero la respuesta es fácil. El Gobierno hubiera lanzado la pelota a otra parte, a la Conferencia Episcopal, a los bomberos, a donde hubiera hecho falta, hasta que alguien dijera sí. Si era una decisión tomada, fruto o no de un pacto, más valía haber dado la cara desde el principio y tener la gallardía de proclamar ante el pueblo español que un Gobierno, cuya existencia se debe a una victoria sobre el comunismo, legalizaba la hoz y el martillo el mismo día que quitaba el yugo y las flechas del edificio de la calle Alcalá, bajo cuya sombra hicieron su carrera relevantes miembros del Gobierno.

Los esquemas de imagen que interactúan en la conformación del dominio origen de la metáfora imago-esquemática troncal LA LEGALIZACIÓN DEL PCE ES UN GOL EN NUESTRA PROPIA PORTERÍA y de las que de ella se derivan (LA ESTRATEGIA DEL GOBIERNO ES ECHAR BALONES FUERA, LA ESTRATEGIA DEL GOBIERNO ES JUGAR A OTRA BANDA, LA LEGALIZACIÓN DEL PCE ES UN BALÓN EN JUEGO) son los siguientes: primero, OBJETO; segundo, FUERZA 
y su subsidiario posibilitación; y, finalmente, PROCeso o movimiento. Tales esquemas de imagen, como demostraremos, construyen metafóricamente la secuencia de acciones ejecutadas por el Gobierno de Suárez para lograr la legalización del PCE, narrada por la publicación, según hemos señalado, como una traición premeditada y muy elaborada.

En el editorial, el esquema de imagen овJето se comporta como un patrón cognitivo básico ${ }^{2}$ que permite al productor textual razonar sobre entidades de naturaleza más inaprensible como si fueran realidades tangibles y tridimensionales, familiares, además, para el lector (portería, balón, pelota). Junto con este patrón cognitivo básico hallamos, como ya hemos dicho, el esquema de imagen FUerza y su subsidiario POSIBILITACIÓN, entendidos ambos en términos de la lógica del camino y generadores de una serie de correspondencias (Peña 1999, 2008): una direccionalidad, que se proyecta sobre las intenciones del gobierno; una trayectoria de movimiento, que se identifica con las consultas hechas a las instituciones judiciales; y un destino, esto es, la inscripción del PCE en el Registro de Asociaciones Políticas.

Naturalmente, el logro de esta meta solo será posible mediante la retirada de los obstáculos que permiten alcanzar el objetivo. En nuestro caso, el obstáculo que ha de vencer el Gobierno es, precisamente, la oposición y el posible levantamiento de los inmovilistas, que se niegan a la legalización del partido. El Gobierno salva este escollo enviando la cuestión, el balón o la pelota, a las distintas instancias que dieron su conformidad a los nuevos estatutos del PCE — condición impuesta por Suárez para una posible legalización—, esto es, el Tribunal Supremo y el Fiscal del Reino o, en clave de hipérbole y con una evidente intención deslegitimadora, "a la Conferencia Episcopal, a los bomberos, a donde hubiera hecho falta, hasta que alguien hubiera dicho sí".

En el marco fuente, estas actuaciones encaminadas a alcanzar el destino pretendido se conceptualizan también, como ya hemos señalado, con el esquema de imagen PROCESO (Peńa 2008), que significa movimiento a lo largo del camino, y que se proyecta en el dominio meta sobre las distintas acciones (echar, pasar, jugar, lanzar, devolver), los distintos pasos, que llevan a cabo el presidente y las instituciones del Estado consultadas para recorrer la senda que conducirá a la meta, la legalización del Partido Comunista.

Las metáforas conceptuales LA ESTRATEGIA DEL GOBIERNO ES ECHAR BALONES FUERA Y LA ESTRATEGIA DEL GOBIERNO ES JUGAR A OTRA BANDA aportan significados claros para cualquier aficionado al fútbol: cuando un equipo se encuentra en una situación comprometida y está rodeado por jugadores del equipo contrario, puede emplear la táctica de lanzar el balón enviándolo a la otra banda o fuera del campo. Estos significados, manipulados por el sesgo ideológico del autor del editorial, se proyectan sobre el marco meta en las maniobras con las que Suárez gestiona su intención de legalizar el PCE opacando su responsabilidad y haciendo creer a la ciudadanía, como ya hemos dicho, que tal decisión no es fruto

\footnotetext{
2 Santibáńez (2002) también ha demostrado que el esquema de imagen овјето puede actuar de forma totalmente independiente en la formación de metáforas; Peña (2008), en cambio, afirma que se trata de un patrón subsidiario o dependiente de otros.
} 
de su voluntad sino el resultado lógico de los dictámenes de las instancias consultadas, responsables aparentes de dar "pase de libre circulación", en palabras del editorial, al PCE en la vida política y social española.

A pesar de que la inscripción del Partido Comunista en el Registro de Asociaciones Políticas constituye un procedimiento puramente administrativo, el presidente Suárez pretende que sean las altas instancias judiciales las que sustenten su determinación, fruto de sus conversaciones secretas con Carrillo y de los cambios experimentados por el citado partido tanto en sus manifestaciones públicas ${ }^{3}$ como en la redacción de sus nuevos estatutos.

Con este propósito, Suárez recorre el siguiente camino: pasa la solicitud de inscripción hecha por el PCE, el balón (LA LEGALIZACIÓN DEL PCE ES UN BALÓN EN JUEGO), al Tribunal Supremo, que devuelve la pelota con el argumento de que en este asunto no tiene jurisdicción. Como en esta banda la táctica no logra el objetivo, no mete el gol, el Gobierno lanza la cuestión a la otra banda, el balón ha ido al Fiscal del Reino (LA ESTRATEGIA DEL GOBIERNO ES ECHAR BALONES FUERA Y LA ESTRATEGIA DEL GOBIERNO ES JUGAR A OTRA BANDA), que responde en el sentido esperado por Suárez. Según recoge El País en su edición del 10 de abril de 1977, el informe del Fiscal alega que de los nuevos estatutos del PCE no se deprende "manifestación que determine la incriminación del partido en cualquiera de las formas de asociación ilícita que define el artículo 172 del Código Penal" y que, consecuentemente, su inscripción en el Registro de Asociaciones Políticas es competencia exclusiva de la Administración. Finalmente, el Ministerio de la Gobernación dispone aprobar la inscripción de la asociación denominada Partido Comunista de España en el Registro.

No todas las metáforas imago-esquemáticas presentan la misma conjunción de patrones cognitivos básicos en sus dominios de partida. Tal es el caso de la proyección LA PORTERÍA DEL GOBIERNO ES LA NO LEGALIZACIÓN DEL PCE, subordinada a la metáfora principal, en cuyo marco fuente hallamos un único esquema de imagen: ОвJето.

El gol lo ha metido el gobierno en su propia portería. En la portería que se juró y perjuró que estaba defendida y bien defendida contra la amenaza comunista. ' $\mathrm{Ni}$ comunismo, ni separatismo, ni terrorismo', se había proclamado enfáticamente. El separatismo campa por sus respetos; al comunismo se le ha dado pase de libre circulación y los terroristas van saliendo, pian pianito, a la calle.

En este caso, se establece una correspondencia ontológica entre la portería y la acción que, en opinión de quienes suscriben este texto, el Gobierno debía evitar a toda costa: la legalización del PCE, conceptualizada por El Alcázar como meter un gol en propia portería, "la portería que se juró y perjuró defender". Esta metáfora contiene una clara valoración implícita, fruto de las proyecciones epistémicas (Lakoff \& Johnson 1999; Lakoff

\footnotetext{
${ }^{3}$ Recordemos, en este sentido, que por entonces el PCE atenúa sus ataques a la Monarquía, acepta la bandera española y renuncia al marxismo-leninismo.
} 
1993) que el lector activa ayudado por el cotexto y el contexto cognitivo: la acción de meter un gol en la propia portería traiciona los intereses del equipo para el que se juega y, por ello, descalifica al protagonista de la jugada, en este caso, el presidente del Gobierno.

\subsection{Metáforas no estructurales}

En la metáfora conceptual que presentamos a continuación el dominio fuente proyecta sobre el meta su rasgo quintaesencial con el objetivo "de aislar y dar prominencia, [...], a un elemento que, utilizado referencialmente, puede actuar como representación del dominio completo" (Ruiz de Mendoza 1999; Ruiz de Mendoza \& Pérez 2011).

En nuestro texto, convertido ahora en un espacio de mezcla (Fauconnier 1997), localizamos dos metáforas ${ }^{4}$ de una correspondencia que, sumadas, contribuyen a la narrativa construida por el editorial y crean la identidad discursiva deslegitimadora de aquellos que respaldan la legalización del PCE. La primera de ellas es LOS PARTIDARIOS DE LA LEGALIZACIÓN SON HINCHAS; y la segunda, LOS PARTIDARIOS DE LA LEGALIZACIÓN SON FIERAS. El uso del término hincha 'partidario entusiasta de un equipo deportivo' (DLE 2018) presupone, en el editorial, un juicio de valor que descalifica a quienes muestran su conformidad con las actuaciones llevadas a cabo por el Gobierno. Este propósito descalificador es fruto también de la identificación de estos hinchas con el marco fuente de los animales, que se instancia lingüísticamente a través de una metonimia que establece una correspondencia entre los gritos de alegría de quienes vitorean la jugada y los rugidos de las fieras:

En las gradas ocupadas por sus hinchas (los del Partido y los del Gobierno), el grito de alegría ha sonado como un rugido: ¡Gol!

Mediante esta conceptualización, los que aplauden la maniobra para legalizar al PCE quedan retratados ideológicamente como seres desprovistos de propiedades humanas e identificados con las fieras a través de un rasgo distintivo, el rugido, que constituye la quintaesencia del 'bruto indómito, cruel y carnicero' (DLE 2018).

\footnotetext{
${ }^{4}$ Con un cambio de marco por el que el deporte del fútbol queda reemplazado por la fiesta del carnaval, encontramos en nuestro texto la metáfora LA LEGALIZACIÓN DEL PCE ES UN CARNAVAL. Este dominio fuente, usado convencionalmente para proyectar atributos esenciales como la máscara, el disfraz y la transgresión, actúa como procedimiento para calificar a Suárez a través de emociones y juicios; por un lado, la máscara y el disfraz representan el engaño, la actuación encubierta del Presidente y su táctica de diluir su propia responsabilidad implicando a las instituciones judiciales; la transgresión, por su parte, se proyecta sobre una decisión que para el autor y sus potenciales lectores sitúa al Presidente y a su Gobierno en el bando enemigo:
}

Estamos en un país libre y cada ciudadano, ministro o jornalero, tiene derecho a elegir el símbolo que quiera. Pero debe hacerlo a cara descubierta, sin arroparse bajo la toga de los juristas.

De todas formas, el carnaval ha terminado. Frente a la amenaza comunista el pueblo español ya sabe dónde está cada uno. Quién es el amigo y quién el enemigo. 
Se trata de una metáfora ontológica (Ruiz de Mendoza y Pérez 2011), que cumple una doble función estratégica: degradar a los partidarios, a los hinchas, a un nivel inferior de la Gran Cadena del Ser (Lovejoy 1936; Lakoff \& Turner 1989), el de los animales; y, al mismo tiempo, explicar su conducta en términos del comportamiento de las bestias. Con ella, el editorial, haciendo uso de una estrategia ampliamente utilizada en el discurso político para representar negativamente las actuaciones del adversario, crea una clara polarización entre el endogrupo y el exogrupo. Como asevera Chilton:

Delegitimization can manifest itself in acts of negative other-presentation, acts of blaming, scapegoating, marginalising, excluding, attacking the moral character of some individual or group, attacking the communicative cooperation of the other, attacking the rationality and sanity of the other. The extreme is to deny the humanness of the other (Chilton 2004: 47).

\section{Conclusiones}

Mediante el análisis del editorial de El Alcázar publicado el 11 de abril de 1977, en el que el periódico se pronuncia contra la legalización del PCE, hemos comprobado que la metáfora se comporta como un potente instrumento cognitivo que permite al escritor narrar y valorar unos acontecimientos difícilmente aceptables para sus lectores. Con esta herramienta, el autor conecta con las prácticas sociales y con los modelos cognitivos de su audiencia, deslegitima la decisión del Gobierno y, con ello, crea una identidad negativa de su adversario.

Como hemos mostrado, el editorial se vertebra alrededor de una metáfora continuada construida a partir del marco fuente DEPORTE y, más concretamente, del submarco fútвol, modelo mental de gran aceptabilidad social y, por ello, muy frecuente en el discurso español. Tal submarco 3/4con sus actores, con su distribución de roles y con la evaluación moral las intenciones, acciones y objetivos de sus protagonistas $3 / 4$ genera una narrativa cuyo hilo argumental es la historia de una traición calculada. Nos referimos, como hemos repetido, a los sucesos que culminan en la noticia inesperada y casi inverosímil para los españoles, sobre todo para los ultraconservadores, de la inscripción del PCE en el Registro de Asociaciones Políticas.

En el texto, este acontecimiento político y social se relata como un partido de fútbol que se salda con la derrota del equipo del que forman parte El Alcázar y sus lectores porque uno de sus jugadores, el Gobierno, mete el gol en su propia portería de forma absolutamente intencionada y valiéndose de estratagemas. Este submarco se reproduce en distintas proyecciones que responden a la distinción entre metáforas estructurales y no estructurales. Todas ellas se subordinan a una metáfora principal, troncal, LA LEGALIZACIÓN DEL PCE ES UN GOL EN NUESTRA PROPIA PORTERÍA.

En la construcción tanto de esta metáfora troncal como de buena parte de las analogías que a ella se subordinan hemos descubierto una pauta recurrente: la configuración 
del dominio origen mediante cuatro esquemas de imagen: OBJETO, FUERZA, POSIBILITACIÓN, y PROCESO o MOVIMIENTO. Tales esquemas de imagen construyen metafóricamente la secuencia de acciones ejecutadas por el Gobierno de Suárez para lograr la legalización del PCE. Así, las consultas que hace el presidente a las distintas instancias judiciales se conceptualizan, de acuerdo con el submarco elegido, en una serie de analogías, cuya interpretación va de la mano, como hemos mostrado, del contexto lingüístico y del cognitivo: LA ESTRATEGIA DEL GOBIERNO ES ECHAR BALONES FUERA, LA ESTRATEGIA DEL GOBIERNO ES JUGAR A OTRA BANDA, LA LEGALIZACIÓN DEL PCE ES UN BALÓN EN JUEGO.

Sin embargo, no todas las metáforas imago-esquemáticas derivadas de la troncal presentan la misma conjunción de patrones cognitivos básicos en sus marcos fuente. Tal es el caso de la proyección LA PORTERÍA DEL GOBIERNO ES LA NO LEGALIZACIÓN DEL PCE, en cuyo dominio de partida hallamos un único esquema de imagen: овJETo. Esta metáfora constituye, sin duda, otra evidencia de que, con su discurso, el editorial de El Alcázar se amolda al modelo cognitivo de sus lectores, contrarios a la legalización, quienes habían dado por supuesto que el presidente del Gobierno, habida cuenta de sus antecedentes, se estaba aplicando a la defensa de su misma portería, proyectada sobre la no legalización.

Por otra parte, junto a estas metáforas, nuestro corpus contiene también analogías cuyo dominio fuente no se corresponde con un esquema de imagen. Tal es el caso de las dos metáforas de una sola correspondencia localizadas: la primera de ellas, que identifica a los partidarios de la legalización con los hinchas (LOS PARTIDARIOS DE LA LEGALIZACión SON HINCHAS), adquiere un propósito descalificador cuando se suma a la segunda de estas proyecciones, LOS PARTIDARIOS DE LA LEGALIZACIÓN SON FIERAS, que los degrada al nivel de las bestias y que acentúa la polarización entre el endogrupo y el exogrupo.

En definitiva, el análisis pormenorizado de las metáforas perfiladas cobre el marco del deporte y, particularmente, del fútbol pone al descubierto la afirmación de Van Dijk (2006) en el sentido de que el discurso político de los medios de comunicación construye una narrativa que impone una determinada comprensión de la realidad acorde con el modelo mental que comparten con sus potenciales lectores.

\section{OBRAS CITADAS}

Beer, Francis \& Christ'l De Landtsheer. 2004. "Metaphors, Politics, and World Politics". En: Francis Beer y Christ'l. De Landtsheer, Eds. Metaphorical World Politics. Michigan: University Press. 5-52.

Casero Ripollés, Andreu. 2004. "Informando del otro: estrategias del discurso periodístico en la construcción de la imagen de los inmigrantes". Sphera Pública 4: 221-236. . 2007. "Discurso mediático, inmigración e ilegalidad: legitimar la exclusión a través de la noticia". En: Ricard Zapata-Barrera y Teun A. Van Dijk, Eds. Discurso sobre la inmigración en España: los medios de comunicación, los parlamentos y las administraciones. Barcelona: Fundación CIDOB. 69-92. 
Charteris-Black, Jonathan. 2004. Corpus approaches to critical metaphor analysis. England: Palgrave-MacMillan.

Chilton, Paul. 1996. Security Metaphors: Cold War Discourse from Containment to Common House. New York: Peter Lang. 2004. Analysing Political Discourse. Theory and Practice. London and New York: Routledge. . 2005. "Missing Links in Mainstream CDA". En: Ruth Wodak y Paul Chilton, Eds. A New Agenda in (Critical) Discourse Analysis. Amsterdam: Benjamins. 19-51.

Croft, William \& D. Alan Cruse. 2004. Cognitive Linguistics. Cambridge: Cambridge University Press.

Díaz-Peralta, Marina. 2016. "Metáforas de la estructura del evento en el discurso político español. Franquismo y nacionalcatolicismo cuarenta años después de la Transición". En: Henry Hernandez-Bayter et al., eds. La transition spagnole 40 ans après. Quels enjeux, quels acquis, quels enseignements? Paris: L'Harmattan. 13-29. . 2018. "Metaphor and ideology: Conceptual structure and conceptual content in Spanish political discourse". Discourse \& Communication 12 (2): 128-148.

Fauconnier, Gilles. 1997. Mappings in thought and language. Cambridge: Cambridge University Press.

Fillmore, Charles J. 1985. "An alternative to checklist theories of meaning". En: C. Cogen et al., Eds. Proceedings of the first annual meeting of the Berkeley Linguistics Society. Berkeley: Berkeley Linguistics Society. 123-1231. . 2006. "Frame semantics". En: Dirk Geeraerts, Ed. Cognitive Linguistics: Basic Readings. New York: Mouton de Gruyter. 373-400.

Fillmore, Charles J. y Beryl T. Atkins. 1992. "Toward a frame-based lexicon: the semantics of RISK and its neiborghs". En A. Lehrer y E. Feder, Eds. Frames, fields and contrasts: new essays in semantics and lexical organization. Hillsdale: Lawrence Erlbaum Associates. 75-102.

Fisher, Walter R. 1984. "Narration as a human communication paradigm: the case of public moral argument". Communication Monographs 51 (1): 1-22. . 1985. "The narrative paradigm: an elaboration". Communication Monographs 52 (4): 347-367.

Glenister, Kathlee. 2004. "Texturing the narrative paradigm: Folklore and communication". Communication Quarterly 52 (2): 129-142.

Grossi, Giorgio. 2004. L'opinione pubblica. Roma-Bari: Laterza

Hart, Christopher. 2008. "Critical discourse analysis and metaphor: toward a theoretical framework". Critical Discourse Studies 5 (2): 91-106.

Johnson, Mark. 1987. The body in the mind: The bodily basis of meaning, imagination, and reason. Chicago: University Press.

. 1993. Moral imagination: Implication of cognitive science for ethics. Chicago: University Press.

Lakoff, George. 1987. Women, fire, and dangerous things. Chicago: Chicago University 
Press.

. 1993. "The contemporary theory of metaphor". En: Andrew Ortony, Ed. Metaphor and thought. New York: Cambridge University Press. 202-251.

. 2002. Moral politics: How liberals and conservatives think. Chicago: University Press.

. 2004. Don't think of an elephant! Know your values and frame the debate. Vermont: Chelsea Green Publishing.

. 2008. The political mind. Why you can't understand 21st-century politics with an 18th-century brain. New York: Penguin.

Lakoff, George \& Mark Johnson. 1980. Metaphors we live by. Chicago and London: University of Chicago Press.

Lakoff, George \& Mark Johnson. 1999. Philosophy in the flesh: The embodied mind and its challenge to Western thought. New York: Basic Books.

Lakoff, George \& Mark Turner. 1989. More than cool reason. Field guide to poetic metaphor. Chicago and London: University of Chicago Press.

Langacker, Ronald. 1987. Foundations of cognitive grammar. Stanford: University Press. 2 vols.

Le Guern, Michael. 1976. La metáfora y la metonimia. Madrid: Cátedra.

Lovejoy, Arthur. 1936. The great chain of being: A study of the history of an idea. Cambridge, Mass.: Harvard University Press.

MacIntyre, Alasdair. 1981. After virtue. London: Duckworth.

McEntee-Atalianis, Lisa. (2011). "The role of metaphor in shaping the identity and agenda of the United Agency: the imagining of an international community and international threat". Discourse \& Communication 5 (4): 393-412.

. 2013. Stance and metaphor: Mapping changing representations of (organizational) identity. Discourse \& Communication 7 (3): 319-338.

Meyer, Michael. 2001. "Between theory, method and politics: positioning of the approaches to CDA”. En: Ruth Wodak y Michael Meyer. Eds. Methods of Critical Discourse Analysis. London: SAGE. 14-31.

Millán, J. A. \& S. Narotzky. 1986. "Introducción a la traducción española de G. Lakoff y M. Johnson”. Metáforas de la vida cotidiana. Madrid: Cátedra.

Montero, Mercedes; Jordi Rodríguez-Virgili \& Carmela García-Ortega. 2008. "La construcción mediática de la comunidad política. La prensa en la transición española a la democracia”. Palabra Clave 11 (2): 293-309.

Muñoz Alonso, Alejandro. 1986. "Golpismo y terrorismo en la transición democrática española”. Reis 36: 25-33.

Musolff, Andrea. 2009. "Metaphor scenarios in public discourse". Metaphor and Symbol 21 (1): 23-38.

Paivio, Allan. 1979. "Psychological process in the comprehension of metaphor". En: Andrew Ortony. Ed. Metaphor and thought. New York: Cambridge University Press. $150-171$. 
Peña Cervel, Sandra. 1999. "Subsidiarity relationships between image-schemas: An approach to the force schema”. Journal of English Studies 1: 187-207.

. 2008. "Dependency systems for image-schematic patterns in a usage-based approach to language". Journal of Pragmatics 40: 1041-1066.

Pinero-Pinero, Gracia; Marina Díaz-Peralta \& María Jesús García-Domínguez. 2014. "Metaphors of irregular immigration in the Spanish language press in the United States". Studia Neophilologica 86 (1): 51-65

Piñero Piñero, Gracia \& John Moore. 2014. "Metáforas legitimadoras del inmigrante irregular en la prensa norteamericana en lengua española”. Onomázein 30: 190-207.

Prego, V. 2002. Sábado Santo Rojo. 25 aniversario de la legalización del PCE (II) [en línea]. Disponible en: http://www.elmundo.es/especiales/2002/04/nacional/sabadorojo/lunes.html

Real Academia de la Lengua. 2018. Diccionario de la lengua española. Madrid: EspasaCalpe [en línea]. Disponible en: http://dle.rae.es

Ruiz de Mendoza Ibáñez, Francisco José. 1999. "The role of cognitive mechanisms in making inferences". Journal of English Studies 1: 237-255.

. 2000. "The role of mappings and domains in understanding metonymy". En: Antonio Barcelona. Ed. Metaphor and metonimy at the crossroads. Berlin: Mouton de Gruyter. 109-132.

Ruiz de Mendoza Ibáñez, Francisco José \& José Luis Otal Campo. 2002. Metonymy, grammar, and communication. Granada: Comares.

Ruiz de Mendoza Ibáńez, Francisco José \& Lorena Pérez Hernández. 2011. "The contemporary theory of metaphor: Myths, developments, and challenges". Metaphor and Symbol 26: 1-25.

Santibáñez, Francisco 2002. "The object image-schema and other dependent schemas". Atlantis 24 (2): 183-201.

Steen, Gerard et al. 2010. Method for linguistic metaphor identification. Amsterdam: John Benjamins.

Stefanowitsch, Anatol. 2006. "Corpus-based approaches to metaphor and metonymy”. En: Anatol Stefanowitsch \& Stefan Gries. Eds. Corpus-based approaches to metaphor and metonymy. Berlin: Mouton de Gruyter. 1-16.

Turner, Mark. 1991. Reading minds: The study of english in the age of cognitive science. Princeton: Princeton University Press.

Van Dijk, Teun. A. 1998. Ideology. A multisdisciplinary approach. London: SAGE. . 2002. "Political discourse and political cognition". En: Paul Chilton \& Christina Schäffner. Eds. Politics as text and talk. Analytical approaches to political discourse. Amsterdam: John Benjamins. 204-236 . 2005. "Ideología y análisis del discurso". Utopía y Praxis Latinoamericana 10 (29): $9-36$. 2006. "Discourse and manipulation”. Discourse \& Society 17 (2): 359-383. 2011. Sociedad y discurso. Cómo influyen los contextos sociales sobre el texto y la con- 
versación. Barcelona: Gedisa.

Zugasti Azagra, Ricardo. 2008. "La prensa como actor colectivo en la transición española a la democracia. El ejemplo de los dos editoriales conjuntos de 1977 ". Revista RE 2 (4): 27-37.

\section{Textos periodísticos citados}

$A B C$. 1977. "La legalización del Partido Comunista: las razones de nuestra discrepancia" [en línea]. Disponible en: http://lahemerotecadelbuitre.com/piezas/sabado-santorojo-el-gobierno-suarez-legaliza-el-pce-partido-comunista-espana-santiago-carrilloalejo-garcia/

El Alcázar. 1977. "Gol” [en línea]. Disponible en: http://lahemerotecadelbuitre.com/piezas/sabado-santo-rojo-el-gobierno-suarez-legaliza-el-pce-partido-comunista-espanasantiago-carrillo-alejo-garcia/

El Pais. 1977. "El Partido Comunista ya es legal" [en línea]. Disponible en: http://lahemerotecadelbuitre.com/piezas/sabado-santo-rojo-el-gobierno-suarez-legaliza-el-pcepartido-comunista-espana-santiago-carrillo-alejo-garcia/

El Pais. 1977. "No hay ilicitud penal en las actividades del PCE” [en línea]. Disponible en: http://elpais.com/diario/1977/04/10/espana/229471203_850215.html

Informaciones. 1977. "Los comunistas dentro de la ley" [en línea]. Disponible en: http:// lahemerotecadelbuitre.com/piezas/sabado-santo-rojo-el-gobierno-suarez-legaliza-elpce-partido-comunista-espana-santiago-carrillo-alejo-garcia/ 